\title{
Immunohistochemical Expression of BRCA1 Protein, ER, PR and Her2/neu in Breast Cancer: A Clinicopathological Study
}

\author{
Israa A Hussein ${ }^{1}$, Shatha Th Ahmed ${ }^{2 *}$, Ameer D Hameedi ${ }^{1}$, Rana Z Naji ${ }^{3}$, Layth \\ Alharbawi ${ }^{4}$, Muzahm Alkhaytt ${ }^{5}$, Intisar S Pity ${ }^{6}$
}

\begin{abstract}
Breast cancer is a heterogeneous hormone-dependent disease. Potential prognosis depends on the clinicopathological evaluation and assessment of other prognostic indicators. The detection of the oestrogen Receptor (ER), Progesterone Receptor (PR), Human epidermal growth factor receptor 2 (Her2/neu) and BRCA1 oncoprotein is pivotal for prognostic evaluation and to choose the appropriate post-surgical adjuvant therapy beside selecting the proper candidate for genetic counselling. Objectives: To detect the immunoexpression of the BRCA1 oncoprotein in mammary invasive ducal carcinoma and its association with the prognostic markers (ER, PR and Her2/neu hormonal receptors) and other clinicopathological parameters to improve the patients' treatment plans. Methods: A cross-sectional study design including 83 paraffin blocks and histological slides collected from Al-Jumhoori Medical City Teaching Hospital Laboratory in Mosul and the Central Public Health Laboratory in Baghdad between the 1st of January 2010 to the 13th of March 2012 for patients diagnosed with primary invasive ductal breast carcinomas. Immunohistochemistry (IHC) using monoclonal antibodies against ER, PR, Her2/neu receptors and BRCA1 protein was performed via the fully automated immunostaining instrument 'Ventana Benchmark'. Results: BRCA1 protein immunoexpression was detected in $20.5 \%$ of cases. It was significantly high with increasing tumour grade and stage. Although there was a trend of BRCA1 negativity toward negative ER, PR and Her2 receptors, no significant associations were observed with any of these parameters and the patients' age. Conclusion: Altered BRCA1 expression is significantly associated with advanced tumour grade and stage. High number of cases with negative BRCA1 expression showed negative ER, PR and Her2/neu expression.
\end{abstract}

Keywords: Breast cancer- BRCA oncoprotein- immunohistochemistry

Asian Pac J Cancer Prev, 21 (4), 1025-1029

\section{Introduction}

Breast cancer $(\mathrm{BC})$ is one of the most common cancers worldwide and is the second leading cause of death among females in the United States (NBCF, 2018). In Iraq, it constitutes around one-third of the overall registered female tumours (Alwan, 2014). Although the backbone for the treatment of $\mathrm{BC}$ is surgical resection, the dilemma for convenient post-operative adjuvant therapies is still prevalent. In order to decide adjuvant therapies to improve the patients' prognosis and survival rates, a thorough assessment of the clinicopathological state, hormonal testing for (oestrogen, progesterone and Her2/neu) receptors in addition to genetic receptors like BRCA1 and BRCA2 are needed. This can be attributed to the fact that $80 \%$ of women with familial tendency to breast and/or ovarian cancer harbour these highly penetrating mutated genes (Shulman, 2010).
The acronym (BRCA) type 1 or 2 stands for breast cancer susceptibility type 1 (BRCA1) and type2 (BRCA2) which are considered as tumour suppressor genes mapped on chromosome 17q21 and 13q12 respectively (Ripperger et al., 2009). These genes can help in repairing DNA damages and prevent uncontrollable cell growth with the inhibition of ER transcriptional activity in the human mammary tissues (Verma et al., 2018; Archey and Arrick, 2017; Wang and Di, 2014). Studies demonstrated that premenopausal women inheriting BRCA1 or BRCA2 gene might be at a higher risk (4 out of 5) for developing breast and/or ovarian cancer as compared to other females of the same age group (1 out of 8) (NBCF 2018; Antoniou et al., 2003; Malone et al., 2000). To the best of our knowledge, limited studies were performed on BRCA immunoexpression and hormone receptor status in our locality, henceforth, this research was performed to check BRCA1 immunoexpression and its association with ER, 
PR and Her2/neu immunoexpression in infiltrative ductal carcinoma of the breast.

\section{Materials and Methods}

An observational cross-sectional study involving 83 paraffin blocks and slides for female patients proven histologically as infiltrative duct carcinoma (not otherwise specified) were collected from Al-Jumhoori Medical Teaching Hospital laboratory in Mosul and Medical City Teaching Hospital in Baghdad, Iraq for the period from the 1st of January 2010 to the 13th of March 2012. Patient's data with regards to their age, histological type, pathological stage and lymph node involvement were retrieved from their medical reports. The tumour histological grading was decided upon examining the H\&E stained slides microscopically using 'Bloom Richardson's grading system' (tubular formation, nuclear pleomorphism, hyperchromatism and number of mitosis). The tumour stage was reported after examining all microscopic slides of the excised tumour, adjacent tissues and the dissected lymph nodes in addition to the clinic-radiologic data when available. Four $\mu \mathrm{m}$ thick sections of the paraffin-embedded blocks were obtained using the fully automated immunostaining instrument 'VentanaBenchmark' (Dako Denmark, Link 48) for immunohistochemistry (IHC) staining using monoclonal antibodies against ER, PR, Her2/neu receptors (ready to use biogenex kit) as described previously by (Ahmed et al., 2018).

Oestrogen and progesterone receptors were considered positive when $\geq 10 \%$ of cell nuclei were positively stained (Figures 1 and 2). These two markers were tested only on 63 cases. For Her $2 /$ neu testing was limited to 32 cases owing to lack of the kit at the time of the study. Only complete circumferential membranous staining in $>10 \%$ of tumour cells (3+) was considered positive (Figure 3). For BRCA1 immunostaining, Mouse monoclonal anti BRCA1' clone MS110 (USA) was used at a dilution of $1: 10$, then scored as negative (greatly reduced or absent) if the brownish nuclear stain was $<20 \%$ while nuclear staining $>20 \%$ was considered as positive (Figures 4-6) (American society of clinical oncology/college of American pathologist clinical practice guideline update 2013).

\section{Results}

Patient's ages $(n=83)$ ranged between 27 and 75 years $($ mean $=47.1$ years $)$, forty-nine females $(59 \%)$ were premenopausal $(<50$ years of age). Among the 83 participants, BRCA1 oncoprotein expression was negative (reduced or absent) in $66(79.5 \%)$ and positive in the remaining 17 cases $(20.5 \%)$. Although the peak age of BRCA1 negative patients was in the fourth decade, no significant correlation found between BRCA1 expression and different age groups (Table 1). On the other hand, 47 $(56.6 \%)$ of the tumors were in stage III, 34 (41\%) stage II and only two cases were in stage I. Tumor grade 3 was the most frequent $41(49.4 \%)$ cases versus $29(34.9 \%)$ and $13(15.6 \%)$ patients with grade 2 and grade 1 respectively.
Table 1. Correlations between BRCA1 Expression with Different Age Groups of the Study Population

\begin{tabular}{lcccc}
\hline $\begin{array}{l}\text { Age group } \\
\text { (years) }\end{array}$ & $\begin{array}{c}\text { BRCA1 }(+) \\
\text { No. }(\%)\end{array}$ & $\begin{array}{c}\text { BRCA1 }(-) \\
\text { No. }(\%)\end{array}$ & $\begin{array}{c}\text { Total } \\
\text { No. }(\%)\end{array}$ & $\begin{array}{c}p- \\
\text { value }\end{array}$ \\
\hline $20-29$ & $0(0 \%)$ & $4(6.06 \%)$ & $4(4.81 \%)$ & $>0.05$ \\
$30-39$ & $4(23.5 \%)$ & $5(7.57 \%)$ & $9(10.84 \%)$ & \\
$40-49$ & $4(23.5 \%)$ & $32(48.48 \%)$ & $36(43.37 \%)$ & \\
$50-59$ & $6(35.29 \%)$ & $23(34.84 \%)$ & $29(34.9 \%)$ & \\
$60-69$ & $1(5.88 \%)$ & $2(3.03 \%)$ & $3(3.61 \%)$ & \\
$>70$ & $2(11.76 \%)$ & $0(0 \%)$ & $2(2.40 \%)$ & \\
Total & $17(100 \%)$ & $66(100 \%)$ & $83(100 \%)$ & \\
\hline
\end{tabular}

${ }^{1}$, BRCA1 denotes to breast cancer susceptibility type 1 .

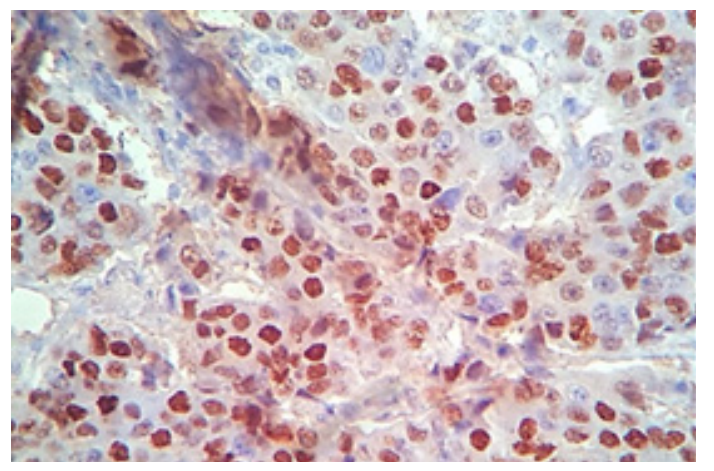

Figure 1. Breast Infiltrative Duct Carcinoma with Positive Nuclear Staining for ER (IHC. X400).

A statistically significant correlation was found between BRCA1 expression with the stage and grade of the tumor (p-value $<0.05$ ) (Table 2). Out of 63 cases tested for ER and PR, 21 (33.33\%) were ER positive and 25 (39.6\%) were PR positive. Out of the $32 \mathrm{Her} 2 /$ neu tested cases, $20(62.5 \%)$ were Her2/neu -ve. Fourteen $(63.63 \%)$ of BRCA1- cases were Her2/neu -ve versus 8 (36.36\%) cases with Her $2 /$ neu +ve. For BRCA+ve cases, 6 out of 10 cases $(60 \%)$ were negative for Her $2 /$ neu and the remaining 4 cases were Her $2 /$ neu + ve. There were no significant correlations between BRCA1 expression and Her2/neu status (Table 3).

Correlating ER/PR subgroups with BRCA1 expression displayed that Phenotype ER-PR- reported in 33 case $(52.38 \%)$ forming the highest incidence, followed by $\mathrm{ER}+\mathrm{PR}+17(26.98 \%)$ and ER-PR+ 9(14.28\%). Phenotype $\mathrm{ER}+\mathrm{PR}-4(6.34 \%)$ registered the lowest incidence. Analysis of the correlation of these subgroups with BRCA1 showed that $29(52.72 \%)$ of the BRCA1-ve

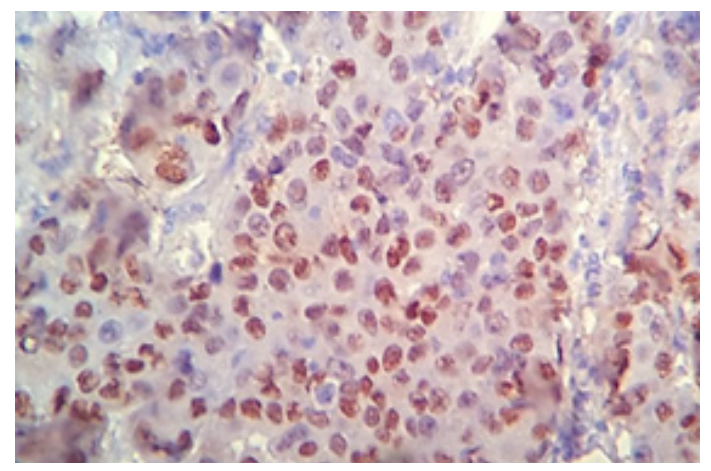

Figure 2. Breast Infiltrative Duct Carcinoma with Positive Nuclear Staining for PR (IHC. X400). 
DOI:10.31557/APJCP.2020.21.4.1025

Immunohistochemical Expression of BRCA1 Protein, ER, PR and Her2/neu in Breast Cancer. A Clinicopathological Study

Table 2. Correlations between BRCA1 Expression with Tumour Stage and Grade

\begin{tabular}{ccccc}
\hline & $\begin{array}{c}\text { BRCA1 }(+) \\
\text { No. }(\%)\end{array}$ & $\begin{array}{c}\text { BRCA1 (-) } \\
\text { No. }(\%)\end{array}$ & $\begin{array}{c}\text { Total } \\
\text { No. }(\%)\end{array}$ & $p$-value \\
\hline Stage & & & & \\
I & $0(0.0)$ & $2(3.0)$ & $2(2.4)$ & $<0.05^{*}$ \\
II & $3(17.6)$ & $31(47.0)$ & $34(41.0)$ & \\
III & $14(82.4)$ & $33(50.0)$ & $47(56.6)$ & \\
IV & $0(0.0)$ & $0(0.0)$ & $0(0.0)$ & \\
Total & $17(100.0)$ & $66(100.0)$ & $83(100.0)$ & \\
Grade & & & & \\
I & $4(23.5)$ & $9(13.6)$ & $13(15.6)$ & $<0.05^{*}$ \\
II & $1(5.8)$ & $27(40.9)$ & $29(34.9)$ & \\
III & $12(70.5)$ & $30(45.45)$ & $41(49.4)$ & \\
IV & $0(0.0)$ & $0(0.0)$ & $0(0.0)$ & \\
Total & $17(100.0)$ & $66(100.0)$ & $83(100.0)$ & \\
\hline
\end{tabular}

${ }^{1}$, RCA1 denotes to breast cancer susceptibility type 1 .

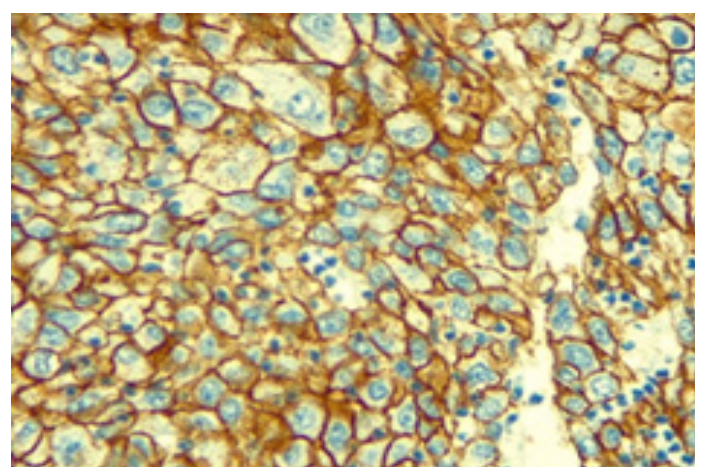

Figure 3. Breast Infiltrative Duct Carcinoma with Positive Membraneous Staining for Her2/neu (IHC. $\mathrm{X} 400)$.

cases were ER-PR-, followed by ER+PR + /BRCA1-ve 15(27.27\%). ER-PR+/BRCA1-ve 7(12.72\%) and the least one was ER+PR-BRCA1-ve 4 (7.27\%) (Table 4). Although the highest number of BRCA1 cases were ER-PR-, there were no significant correlations between BRCA1 and ER, PR status ( $p$-value $>0.05$ ).

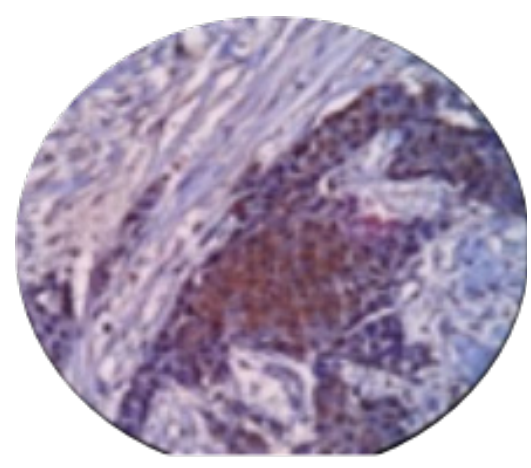

Figure 4. Breast Infiltrative Duct Carcinoma with a Diffuse Nuclear Staining of Weak to Moderate Intensity for BRCA1(IHC. X100).
Table 3. Frequency and Percentage of ER, PR, Her2/neu Expression in BRCA1(+ve) and BRCA1(-ve) Cases

\begin{tabular}{|c|c|c|c|c|}
\hline & $\begin{array}{c}\text { BRCA1 }^{1}(+) \\
\text { No. }(\%)\end{array}$ & $\begin{array}{c}\text { BRCA1 (-) } \\
\text { No. }(\%)\end{array}$ & $\begin{array}{c}\text { Total } \\
\text { No. (\%) }\end{array}$ & $\begin{array}{c}p- \\
\text { value }\end{array}$ \\
\hline \multicolumn{5}{|c|}{ Oestrogen Receptors (ERs) } \\
\hline $\mathrm{ER}^{2}+$ & $2(28.5)$ & $19(33.9)$ & $21(33.33)$ & $>0.05$ \\
\hline ER - & $5(71.4)$ & $37(66.07)$ & $42(66.66)$ & \\
\hline Total & $7(100.0)$ & $56(100.0)$ & $63(100.0)$ & \\
\hline \multicolumn{5}{|c|}{ Progesterone Receptors (PRs) } \\
\hline $\mathrm{PR}^{3}+$ & $3(42.85)$ & $23(41.07)$ & $25(39.6)$ & $>0.05$ \\
\hline PR - & $4(57.15)$ & $33(58.93)$ & $38(60.4)$ & \\
\hline Total & $7(100.0)$ & $56(100.0)$ & $63(100.0)$ & \\
\hline \multicolumn{5}{|c|}{ Her2/neu Receptors } \\
\hline Her $2 /$ neu $^{4}+$ & $4(40)$ & $8(36.36)$ & $12(37.5)$ & $>0.05$ \\
\hline Her2/neu - & $6(60)$ & $14(63.63)$ & $20(62.5)$ & \\
\hline Total & $10(100.0)$ & $22(100.0)$ & $32(100.0)$ & \\
\hline
\end{tabular}

${ }^{1}$, BRCA1 denotes to breast cancer susceptibility type $1 ;{ }^{2}$, ER denotes to oestrogen receptors; ${ }^{3}$, PR denotes to progesterone receptors; ${ }^{4}$, Her $2 /$ neu denotes to human epidermal growth factor receptor 2 .

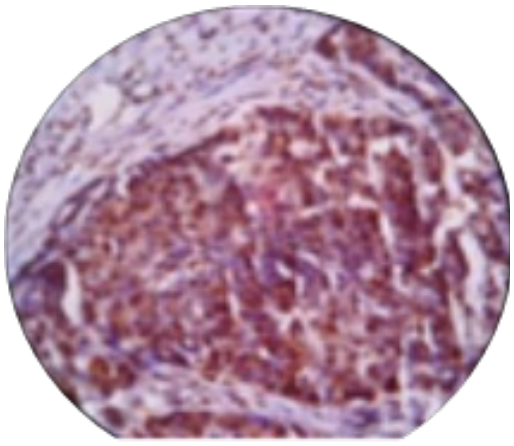

Figure 5. Breast Infiltrative Duct Carcinoma with a Diffuse Nuclear Staining of Moderate Intensity for BRCA1(IHC. X100).

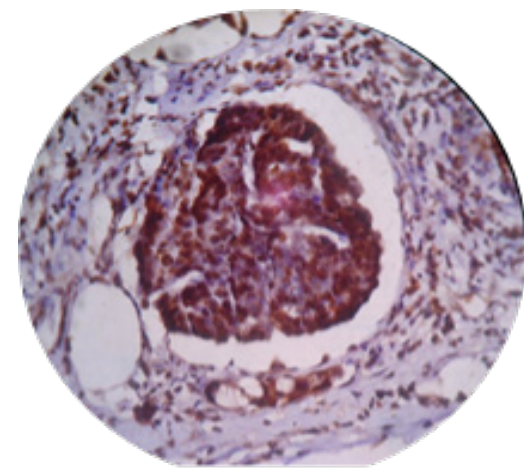

Figure 6. Vascular Invasion with Breast Carcinoma with Nuclear Positivity for BRCA1(IHC,X100).

Table 4. Correlation between ER, PR Subtypes Expression in BRCA1(+) and BRCA1(-) Cases

\begin{tabular}{lcccc}
\hline ER, PR & BRCA1 (+) & BRCA1 (-) & Total & $P-$ \\
subgroups & No. $(\%)$ & No. (\%) & No. (\%) & value \\
\hline ER+PR+ & $2(28.57)$ & $15(27.27)$ & $17(26.98)$ & $>0.05$ \\
ER-PR- & $4(57.14)$ & $29(52.72)$ & $33(52.38)$ & \\
ER+PR- & $0(00 \%)$ & $4(7.27)$ & $4(6.34)$ & \\
ER-PR+ & $1(14.2)$ & $7(12.72)$ & $9(14.28)$ & \\
Total & $7(100.0)$ & $55(100.0)$ & $63(100.0)$ & \\
\hline
\end{tabular}

Asian Pacific Journal of Cancer Prevention, Vol 21 


\section{Discussion}

In the current study using immunohistochemistry, $20.4 \%$ were positively stained for BRCA1 whereas Verma et al. reported $55.6 \%$ nuclear positivity for BRCA1 in their study (Verma et al., 2018). A significant relation between BRCA1 expression and advanced tumour stage was found which coincides with the reports from other researches (Bugrein and Bujassoum, 2016; Ashraf et al., 2011). Additionally, we found that there is a significant association between BRCA1 immunoexpression and graded tumours, mostly grade III (49.4\%). This came in contrast to Yang et al. who demonstrated a significant inverse relationship between BRCA1 expression and histologic grade (Yang et al., 2001). These findings regarding the advanced stage and high grade with BRCA1 expression may reflect the poor prognosis of tumours harbouring this gene mutation (Hedau et al., 2015; Yang et al., 2001).

An interesting finding in the current study was the high frequency of negative ER and PR were observed in BRCA1 negative group. Despite not being statistically significant, a trend of Her2/neu negative BCs toward lack of BRCA1 expression was observed. Ansquer et al. argued that BRCA1 expression was not related to Her2/ neu expression (Ansquer et al., 2005). A considerable number of studies demonstrated negative ER, PR and Her2/neu in familial BC (Yang et al., 2015; Chappuis et al., 2000; Henderson and Patek, 1998). Similar findings have been reported in other literatures (Verma et al., 2018; Kumar et al., 2017; Ashraf et al., 2011).

In the same line, Jóhannsson et al., (1997) observed a significant lower frequency of ER/PR positive expression in BRCA1 tumours using immunohistochemical staining of paraffin sections. However, higher values were reported by others (Geredeli et al., 2019; Huszno et al., 2019; Verma et al., 2018). Such differences could be attributed to the variations in the intra and inter laboratory technical immunostaining methods (Iqbal and Buch, 2016). Other studies reported that the majority of BRCA1-related tumors were grade III invasive ductal cancers and were ER negative regardless of the age and stage of the disease (Krammer et al., 2017). This raises the concept that ER negativity is neither a sequel of younger ages nor the graded tumours at onset of diagnosis, but is an intrinsic character related to BRCA1 positive cancers (Shuen and Foulkes, 2012; Foulkes et al., 2004). Similarly, Tung et al, reported in 2010 that Patients younger than 50 years diagnosed with $\mathrm{BC}$ were significantly more likely to have an ER negative cancer compared with older aged patients (Tung et al., 2010). It is important to mention that considerable percentages of negative ER may be "false negative" due to some technical factors related to inappropriate tissue fixation, antigen retrieval or staining a necrotic areas (Mouttet et al., 2016; Nadji et al., 2005). Moreover, the lack of standardised measurements of grade and ER increases the errors in the results (Foulkes et al., 2004). Furthermore, the cut off point for negativity of ER/ PR in most studies is calculated as immunostained active cells $<10 \%$ whereas the new guidelines consider negative ER/PR as $<1 \%$ (Mouttet et al., 2016).
In conclusion, our study suggests that BRCA1 expression is significantly correlated with an advanced tumour grade and stage. A higher number of cases with negative BRCA1 immunoexpression showed negative ER, PR and Her2/neu expression. These findings raise the importance of identifying molecular profiling of ER, PR, Her2/neu and the state of BRCA1 expression as useful prognostic markers for $\mathrm{BC}$ patients before starting treatment and selecting proper persons for the genetic screening of the mutated BRCA1 oncoprotein.

\section{Acknowledgments}

We would like to thank all the staff in the pathology laboratories in Mosul and Baghdad for their great help in staining and photographing our slides. Also great thanks to the members in the statistical department in Mosul medical college for their help in analyzing our data.

This study was funded by the contributing authors, and was ethically approved.

\section{References}

Ahmed ST, Ahmed AM, Musa DH, et al (2018). Proliferative index (Ki67) for prediction in breast duct carcinomas. Asian Pac J Cancer Prev, 19, 955-9.

Alwan N (2014). Iraqi initiative of a regional comparative breast cancer research project in the Middle East. J Cancer Biol Res, 2, 1006.

Ansquer Y, Mandelbrot L, Lehy T, et al (2005). Expression of BRCA1, HER-1 (EGFR) and HER-2 in sporadic breast cancer and relationships to other clinicopathological prognostic features. Anticancer Res, 25, 4535-41.

Antoniou A, Pharoah PD, Narod S. et al (2003). Average risks of breast and ovarian cancer associated with BRCA1 or BRCA2 mutations detected in case series unselected for family history: A combined analysis of 22 studies. Am J Hum Genet, 72, 1117-30.

Archey WB, Arrick BA (2017). Transactivation of the estrogen receptor promoter by BRCA1. Cancer Cell Int, 17, 33 .

Ashraf M, Jha JK, Mukherjee N, et al (2011). BRCA1 protein expression and its correlation with ER/PR status in sporadic and familial breast cancer in Eastern Indian patients--a hospital based study. J Indian Med Assoc, 109, 873-8.

Bugrein H, Bujassoum SM (2016). Genotype and phenotype correlation of breast cancer in BRCA carriers and non-carriers. Qatar Found Ann Res Conf Proc, 2016, HBPP1674hp://dx.doi.org/10.5339/fare.2016.HBPP1674.

Chappuis PO, Victoria MS, William D (2000). Clinico-pathological characteristics of BRCA1- and BRCA2-related breast cancer. Sem Surg Oncol, 18, 287-95.

Foulkes WD, Metcalfe K, Sun P, et al (2004). Estrogen receptor status in BRCA1- and BRCA2-related breast cancer: The influence of age, grade, and histological type. Clin Cancer Res, 10, 2029-34.

Geredeli C, Yasar N, Sakin A (2019). Germline mutations in BRCA1 and BRCA2 in breast cancer patients with high genetic risk in Turkish population. Available at: https:// www.hindawi.com/journals/ijbc/2019/9645147/[ [Accessed: 19 September 2019].

Hedau S, Batra M, Singh UR, et al (2015). Expression of BRCA1 and BRCA2 proteins and their correlation with clinical staging in breast cancer. J Cancer Res Ther, 11, 58-63.

Henderson IC, Patek AJ (1998). The relationship between prognostic and predictive factors in the management of 
breast cancer. Breast Cancer Res Treat, 52, 261-88.

Huszno J, Kołosza Z, Grzybowska E, et al (2019). BRCA1 mutation in breast cancer patients: Analysis of prognostic factors and survival. Oncol Lett, 17, 1986-95.

Iqbal BM, Buch A (2016). Hormone receptor (ER, PR, HER2/ neu) status and proliferation index marker (Ki-67) in breast cancers: Their onco-pathological correlation, shortcomings and future trends. Med J Dr D Y Patil Univ, 9, 674.

Jóhannsson OT, Idvall I, Anderson D, et al (1997). Tumour biological features of BRCA1-induced breast and ovarian cancer. Eur J Cancer, 33, 362-71.

Krammer J, Pinker-Domenig K, Robson ME et al (2017). Breast cancer detection and tumor characteristics in BRCA1 and BRCA2 mutation carriers. Breast Cancer Res Treat, 163, 565-71.

Kumar M, Sahu RK, Goyal A, et al (2017). BRCA1 promoter methylation and expression - associations with ER+, PR+ and HER2+ subtypes of breast carcinoma. Asian Pac $J$ Cancer Prev, 18, 3293-99.

Malone KE, Dailing JR, Neal C, et al (2000). Frequency of BRCA1/BRCA2 mutations in a population-based sample of young breast carcinoma cases. Cancer, 88, 1393-402.

Mouttet D, Laé M, Caly M, et al (2016). Estrogen-receptor, progesterone-receptor and HER2 status determination in invasive breast cancer. Concordance between immuno-histochemistry and MapQuantTM Microarray Based Assay. PLoS One, 11. Available at: https://www. ncbi.nlm.nih.gov/pmc/articles/PMC4735463/ [Accessed: 19 September 2019].

Nadji M, Gomez-Fernandez C, Ganjei-Azar P, et al (2005). Immunohistochemistry of estrogen and progesterone receptors reconsidered experience with 5,993 breast cancers. Am J Clin Pathol, 123, 21-7.

NBCF (2018). Breast cancer facts: The National Breast Cancer Foundation. Available at: https://www.nationalbreastcancer. org/breast-cancer-facts [Accessed: 19 September 2019].

Ripperger T, Gadzicki D, Meindl A, et al (2009). Breast cancer susceptibility: current knowledge and implications for genetic counselling. Eur J Hum Genet, 17, 722-31.

Shuen A, Foulkes W (2012). Basal-like breast cancercharacteristics, risks, and associations.touch ONCOLOGY. Availableat: https://touchoncology.com/basal-like-breastcancer-characteristics-risks-and-associations/ [Accessed: 19 September 2019].

Shulman LP (2010). Hereditary breast and ovarian cancer (HBOC): Clinical features and counseling for BRCA1 and BRCA2, lynch syndrome, cowden syndrome, and Li-fraumeni syndrome. Obstet Gynecol Clin, 37, 109-33.

Tung N, Wang Y, Collins LC, et al (2010). Estrogen receptor positive breast cancers in BRCA1 mutation carriers: clinical risk factors and pathologic features. Breast Cancer Res, 12, R12.

Verma D, Agarwal K, Tudu S (2018). Expression of breast cancer type 1 and its relation with expression of estrogen receptors, progesterone receptors, and human epidermal growth factor receptor $2 /$ neu in breast carcinoma on trucut biopsy specimens. Indian J Pathol Microbiol, 61, 31.

Wang L, Di LJ (2014). BRCA1 and estrogen/estrogen receptor in breast cancer: Where they interact?. Int J Biol Sci, 10, 566-75.

Yang Q, Sakurai T, Mori I, et al (2001). Prognostic significance of BRCA1 expression in Japanese sporadic breast carcinomas. Cancer, 92, 54-60.

Yang X, Wu J, Lu J, et al (2015). Identification of a comprehensive spectrum of genetic factors for hereditary breast cancer in a chinese population by next-generation sequencing. PLoS One, 10, Available at: https://www.ncbi.nlm.nih.gov/pmc/
articles/PMC4415911/ [Accessed: 5 October 2019].

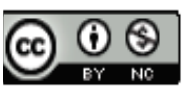

This work is licensed under a Creative Commons AttributionNon Commercial 4.0 International License. 